Review began 12/02/2021 Review ended 12/16/2021 Published 12/21/2021

(c) Copyright 2021

Craig et al. This is an open access article distributed under the terms of the Creative Commons Attribution License CC-BY 4.0. which permits unrestricted use, distribution, and reproduction in any medium, provided the original author and source are credited.

\section{Dissolving Potassium Tablets Masquerading as Concealed Foreign Body Ingestion}

\author{
Michael A. Craig ${ }^{1}$, Carl Kay ${ }^{2}$, Thomas B. Wells ${ }^{3}$, Maurice C. Barnes ${ }^{3}$ \\ 1. Internal Medicine, Brooke Army Medical Center, San Antonio, USA 2. Gastroenterology, Brooke Army Medical \\ Center, San Antonio, USA 3. Gastroenterology, The University of Tennessee Health Science Center, Memphis, USA
}

Corresponding author: Michael A. Craig, craig.michael62@gmail.com

\begin{abstract}
Foreign body ingestion is a common consultation for gastroenterologists. Without knowing the object(s) ingested, the differential diagnosis is broad, especially in patients with underlying mental health conditions, such as uncontrolled bipolar disorder, prior suicide attempts, or recreational drug use. The differential should include substances taken with suicidal intent or for concealment of illicit drugs. Certain foreign objects may require urgent or emergent endoscopic intervention. However, one should also consider benign, iatrogenic causes such as large, radiolucent potassium pills given in the emergency department, which do
\end{abstract} not require further intervention or hospitalization.

Categories: Radiology, Gastroenterology

Keywords: radio-opaque, deliberate foreign body ingestion, potassium chloride, non-intentional self-ingestion, image findings

\section{Introduction}

Foreign body ingestion occurs frequently, and often results in patients seeking care at emergency facilities. Approximately $80 \%$ of ingestions can be managed conservatively, but $10-20 \%$ do require endoscopic intervention [1]. Of note, the subset of foreign objects that are intentionally ingested may have increased need for endoscopic intervention (63-76\%) or surgical intervention (12-16\%) [2,3]. These intentional ingestions can include sharp objects, pieces of glass, razor blades, or packets of illegal drugs. Many complications can arise from accidental or incidental ingestion of foreign bodies, such as complete obstruction of the esophagus, perforation of gastrointestinal viscera, or overdoses of consumed drugs [4]. For this reason, it is important that patients with ingestion are evaluated appropriately with a thorough history and physical examination, imaging, and subspecialty consultation if appropriate. The endoscopic management of ingested objects varies depending on the type of the object, the location of the object, and the timing of ingestion [5]. However, it is important to note that benign medications such as radiopaque tablets given in the emergency department (ED) may not require endoscopy [6,7]. Here we present a case of large potassium tablets mimicking ingestion of potentially lethal objects.

\section{Case Presentation}

A 25-year-old male with untreated bipolar disorder, substance abuse, and a prior suicide attempt was brought to the ED after a motor vehicle collision. He was an unrestrained passenger who was hit by another vehicle. In the trauma ED, he was evaluated for injuries with a computed tomography (CT) scan of his chest, abdomen, and pelvis. During the evaluation by the ED physician, he endorsed suicidal ideation. He was hemodynamically stable with unremarkable labs except for potassium of $2.9 \mathrm{mmol} / \mathrm{L}$. CT scan showed no evidence of traumatic findings, obstruction, free air, or ascites. However, within the stomach there were two rounded hyperdense objects measuring approximately $2.5 \times 1 \times 1 \mathrm{~cm}$, which could represent an ingested foreign body (Figures 1,2). 


\section{Cureus}

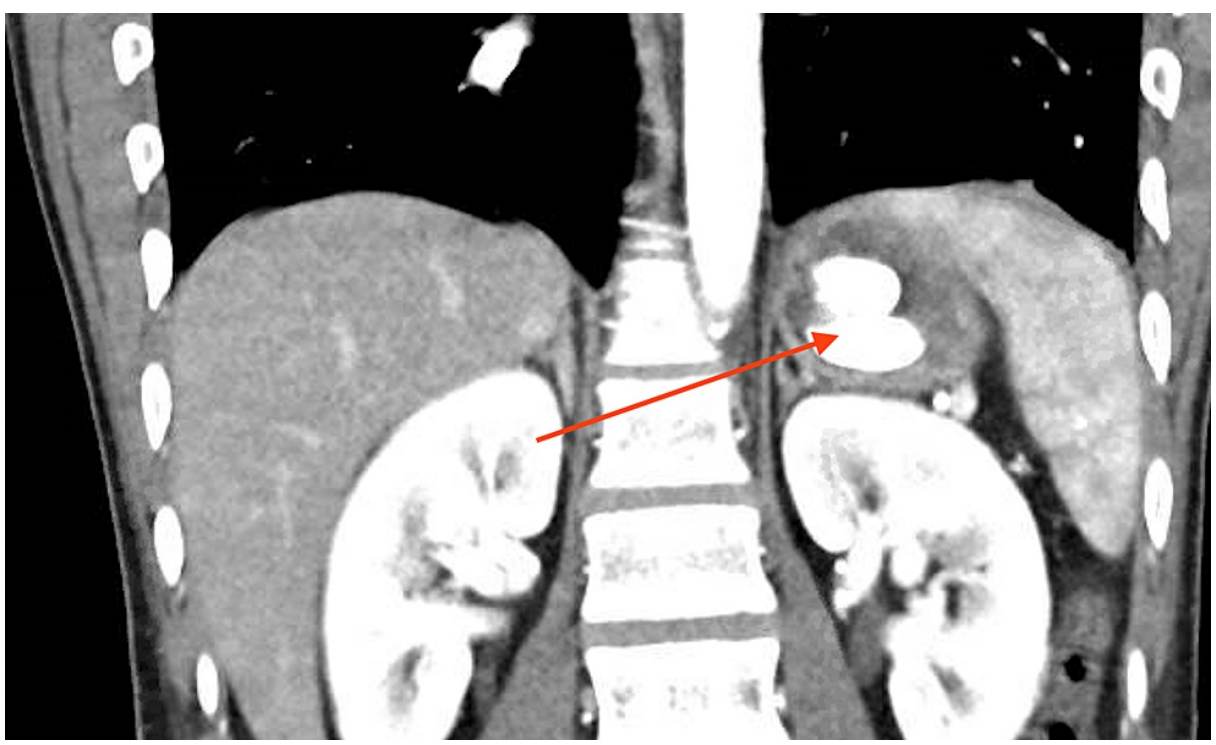

FIGURE 1: Two foreign bodies seen in the stomach on CT scan with red arrow indicating location

$\mathrm{CT}$, computed tomography

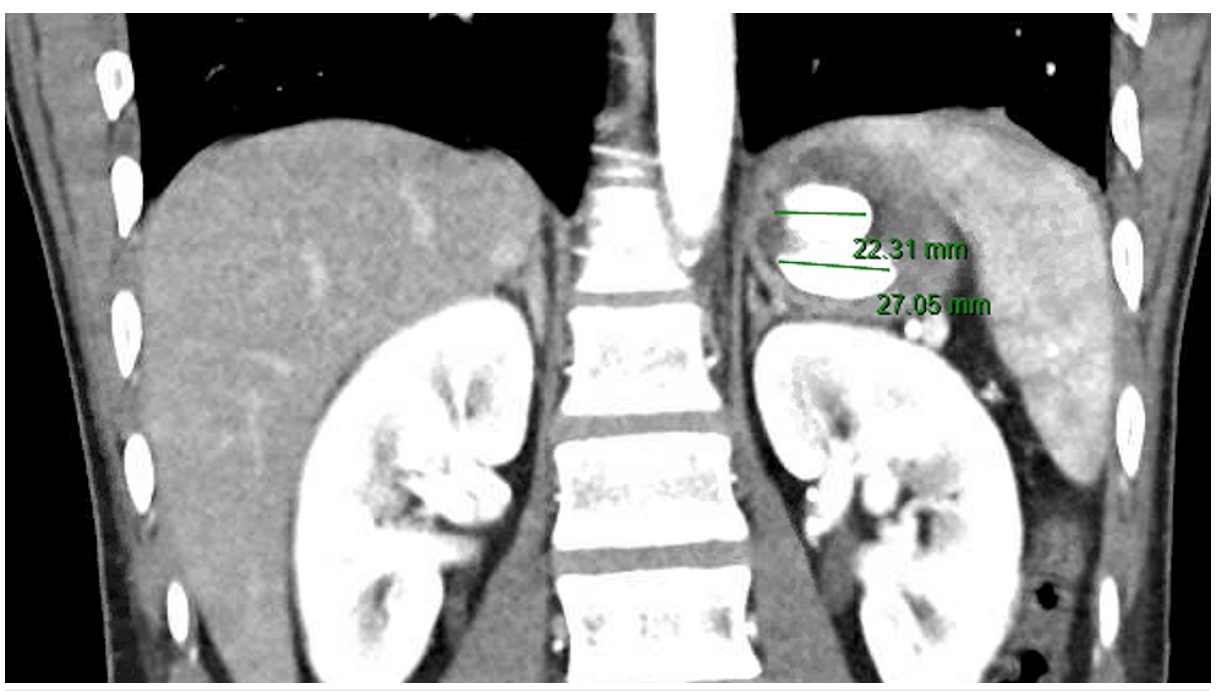

FIGURE 2: Two foreign bodies seen in the stomach on CT, measured via radiology software

$\mathrm{CT}$, computed tomography

Based on these findings, the patient was asked if he had ingested any foreign objects, which he denied. He was admitted for observation due to potential foreign body ingestion and suicidal ideation. Even though the patient denied any intentional ingestion, the leading diagnosis at presentation was ingestion of a package of drugs to conceal it from authorities. Gastroenterology was consulted at this time.

On evaluation by the gastroenterology service, the patient continued to deny any foreign body ingestion. Review of the medication administration log showed he was given two $40 \mathrm{mEq} \mathrm{KCl}$ tablets 10 minutes prior to the CT scan for hypokalemia. After confirming with pharmacy, the $\mathrm{KCl}$ tablets at the institution measured $1 \mathrm{~cm} \times 1 \mathrm{~cm} \times 2 \mathrm{~cm}$ (Figure 3). Repeat abdominal X-rays a few hours after the CT scan showed no signs of a foreign body (Figure 4). The gastroenterology team determined that the foreign objects seen on CT scan were likely the two $\mathrm{KCl}$ tablets he had ingested just minutes before imaging. The patient was evaluated by a mental health provider and deemed safe for discharge by the gastroenterology service and a mental health provider without need for endoscopic intervention. 


\section{Cureus}

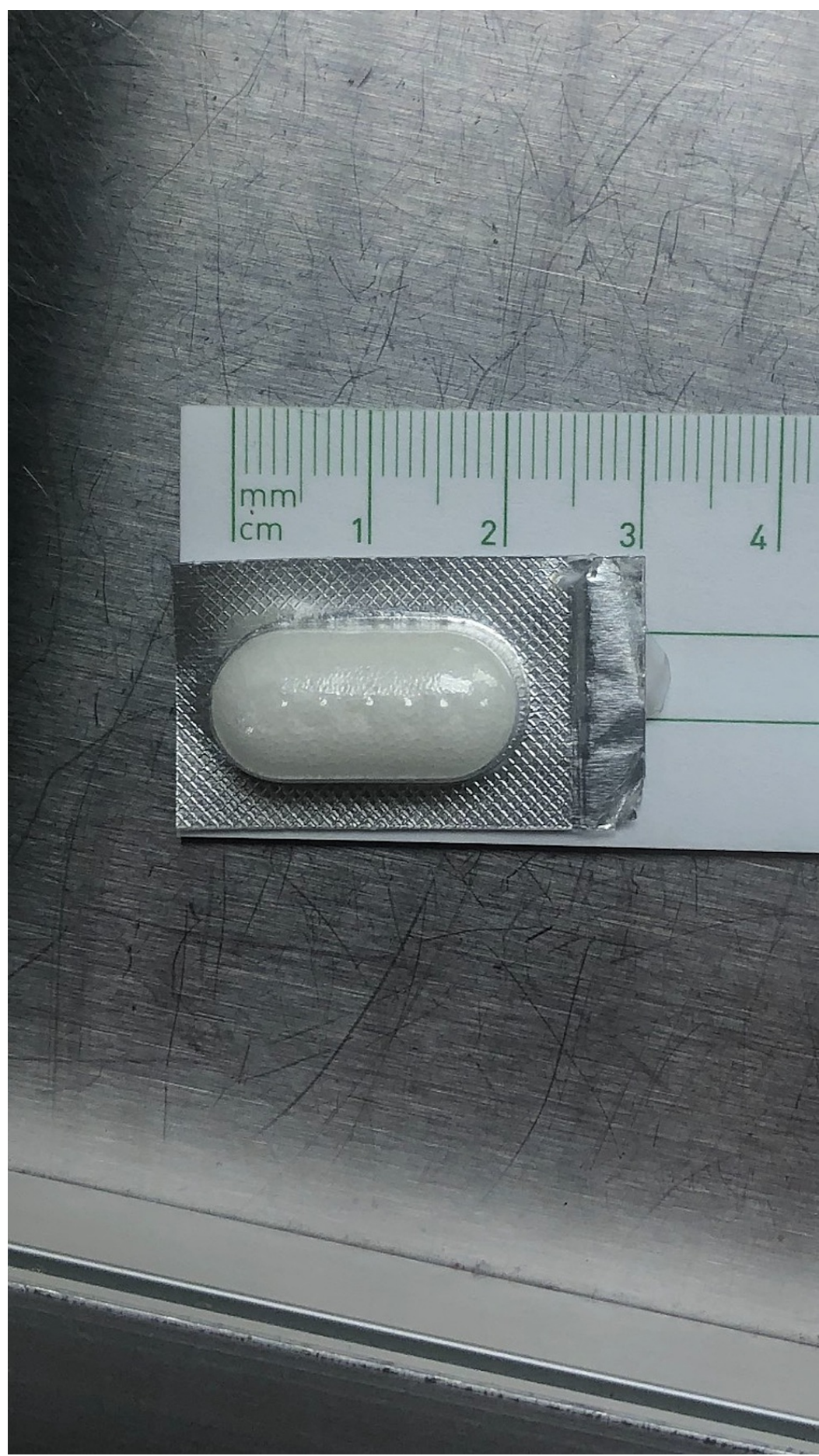

FIGURE 3: The potassium pills carried by the hospital, measuring 20 $\mathrm{mm}$ 


\section{Cureus}

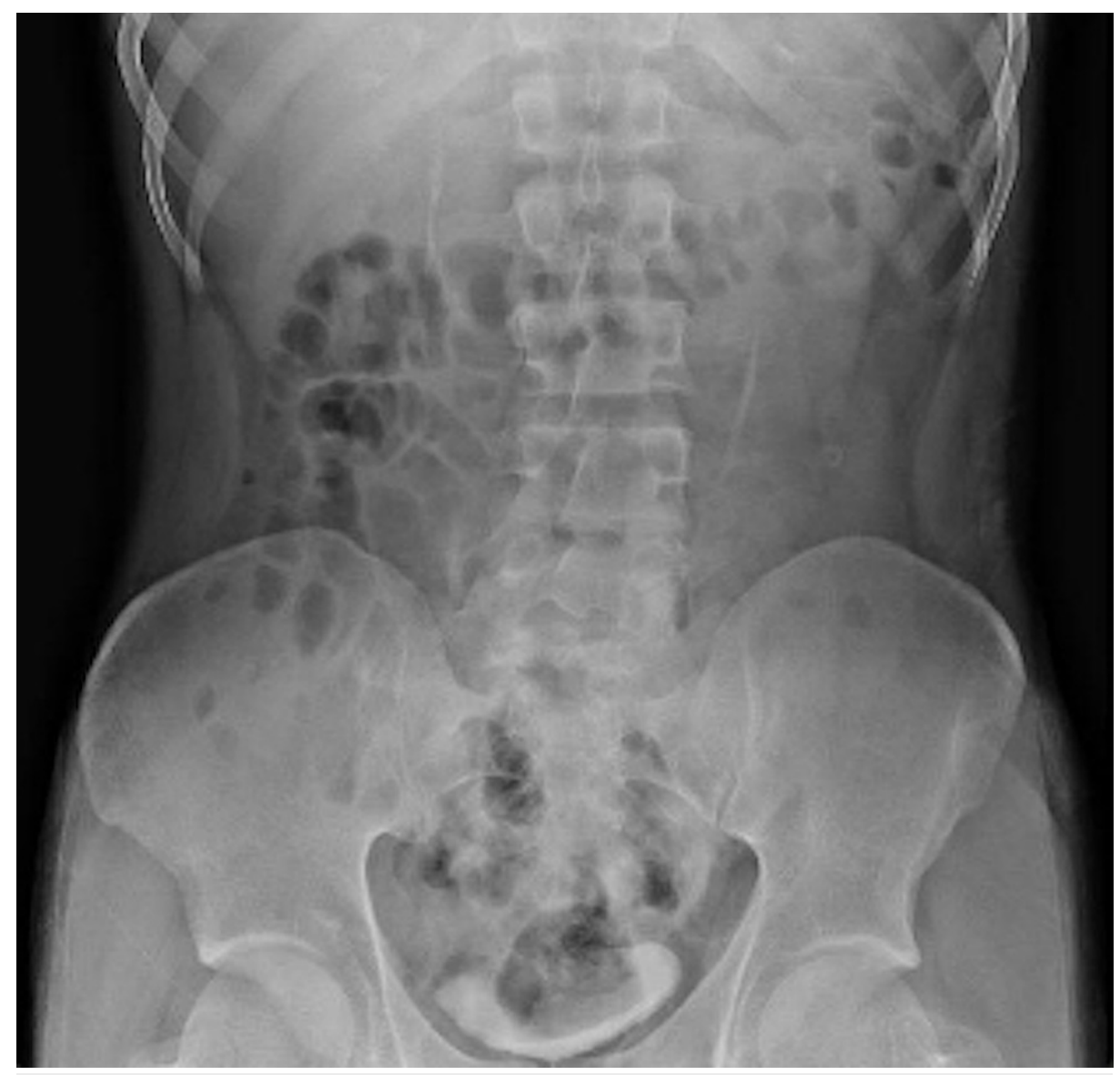

FIGURE 4: Unremarkable abdominal X-ray several hours after foreign bodies were seen on CT

$\mathrm{CT}$, computed tomography

\section{Discussion}

Foreign body ingestion is a reasonable indication for gastroenterology consultation, due to the potential need for endoscopic intervention emergently or urgently. Given the psychiatric comorbidities and possible motive for concealing illicit substances from authorities after the motor vehicle collision, foreign body ingestion was reasonable to include within the initial differential diagnosis in the context of the CT findings [4]. However, given the characteristics of the foreign body, there were no indications for emergent or urgent intervention, as the visualized object was blunt, medium sized, and located within the stomach (Tables 1, 2) [1]. It should also be noted that the European Society of Gastrointestinal Endoscopy Guidelines on Foreign Body Ingestion for 2016 recommend against endoscopic intervention of "drug packing" cases due to the risk of rupture and potential overdose, and recommend surgical referral in cases of packet rupture or obstruction [5]. 


\section{Cureus}

\begin{tabular}{|l|l|l|}
\hline Object type & Location & Timing \\
\hline Sharp-pointed foreign body & Esophagus & Emergent \\
\hline Blunt and small foreign body $(<2 \mathrm{~cm}$ diameter $)$ & Stomach/small bowel & Urgent \\
\hline Blunt and medium foreign body $(2-5 \mathrm{~cm}$ diameter $)$ & Esophagus & Non-urgent \\
\hline Blunt and large foreign body $(>5 \mathrm{~cm}$ diameter $)$ & Stomach/small bowel & Urgent \\
\hline
\end{tabular}

TABLE 1: Management of foreign bodies

Adapted from the 2016 ESGE guidelines [5]

\begin{tabular}{|l|l|}
\hline \hline Type & Examples \\
\hline Blunt & Coin, button, toy, packet of illegal drugs \\
\hline Sharp-pointed & Needle, toothpick, bone, safety pin, glass, razor blades \\
\hline
\end{tabular}

\section{TABLE 2: Categories of ingested objects}

Adapted from the 2016 ESGE guidelines [5]

Various other radiopaque pills can also masquerade as foreign bodies, including heavy metals, iron, and sustained-release medications [6]. Other medications that have been visualized as foreign bodies include theophylline, erythromycin, and multivitamins; of the medications tested in a 1998 Mayo Clinic study, potassium chloride is the most radio-dense (Table 3) [7]. Of note, an ingested potassium pill has also mimicked a hyperdense lesion suggestive of gastric bleeding on CT [8]. Other foreign bodies that can be incidentally found within the gastrointestinal lumen on imaging include bezoars, gallstones, endoscopic capsules, dentures, and batteries [9].

\begin{tabular}{|cc} 
Medication & Rela \\
Potassium chloride & 0.52 \\
Ferrous sulfate & 0.43 \\
Calcium carbonate & 0.35 \\
Theophylline & 0.20 \\
Erythromycin & 0.20 \\
Amoxicillin-clavulanate & 0.20 \\
Clarithromycin & 0.18
\end{tabular}

\section{TABLE 3: Relative radiodensity of various medications}

Calculated by subtracting the background density from the density of the pill at its center. Adapted from Mayo Clinical Proceedings, 1998 [7] 
on CT. Repeat imaging was performed several hours later, which demonstrated complete resolution of the radiopaque objects. No overt passage of foreign bodies occurred in the stool while the patient was admitted. Because endoscopy was not pursued, the $\mathrm{KCl}$ tablets were never directly visualized within the gastric lumen. However, it is reasonable to assume that these tablets were the direct cause of the CT findings.

In summary, the temporal relationship between oral potassium repletion and imaging is an important consideration when confronted with unexpected foreign bodies found on non-invasive imaging studies, especially in patients with a past medical history of psychiatric illness or patients presenting to the hospital after contact with law enforcement.

\section{Conclusions}

Foreign body ingestion is a common cause of emergency room visits. Ingestion of accidental and intentional foreign bodies such as obstructive food boluses, sharp objects, lethal doses of medication, or "drug packing" can cause serious complications requiring the need for emergent or urgent endoscopic or surgical interventions. These complications can include complete esophageal obstruction, perforation of abdominal organs, or overdose, but not every case requires gastrointestinal consultation or procedural intervention. It is possible for benign, radiopaque objects such as potassium chloride tablets to mimic these potentially lethal ingestions. We present this case to make other physicians aware that in the right setting, medication tablets should be considered in the differential for foreign body ingestion. As always, a thorough history from the patient and review of the electronic medical records can help identify these benign cases.

\section{Additional Information \\ Disclosures}

Human subjects: Consent was obtained or waived by all participants in this study. Conflicts of interest: In compliance with the ICMJE uniform disclosure form, all authors declare the following: Payment/services info: All authors have declared that no financial support was received from any organization for the submitted work. Financial relationships: All authors have declared that they have no financial relationships at present or within the previous three years with any organizations that might have an interest in the submitted work. Other relationships: Disclosure statement: The view(s) expressed herein are those of the author(s) and do not reflect the official policy or position of Brooke Army Medical Center, the U.S. Army Medical Department, the U.S. Army Office of the Surgeon General, the Department of the Army, the Department of the Air Force, the Department of Defense, or the U.S. Government.

\section{References}

1. Ikenberry SO, Jue TL, Anderson MA, et al.: Management of ingested foreign bodies and food impactions . Gastrointest Endosc. 2011, 73:1085-91. 10.1016/i.gie.2010.11.010

2. Palta R, Sahota A, Bemarki A, Salama P, Simpson N, Laine L: Foreign-body ingestion: characteristics and outcomes in a lower socioeconomic population with predominantly intentional ingestion. Gastrointest Endosc. 2009, 69:426-33. 10.1016/i.gie.2008.05.072

3. Weiland ST, Schurr MJ: Conservative management of ingested foreign bodies . J Gastrointest Surg. 2002, 6:496-500. 10.1016/s1091-255x(01)00027-0

4. Gill JR, Graham SM: Ten years of "body packers" in New York City: 50 deaths . J Forensic Sci. 2002, 47:843-6.

5. Birk M, Bauerfeind P, Deprez PH, et al.: Removal of foreign bodies in the upper gastrointestinal tract in adults: European Society of Gastrointestinal Endoscopy (ESGE) Clinical Guideline. Endoscopy. 2016, 48:48996. 10.1055/s-0042-100456

6. Savitt DL, Hawkins HH, Roberts JR: The radiopacity of ingested medications . Ann Emerg Med. 1987, 16:3319. 10.1016/s0196-0644(87)80182-8

7. Florez MV, Evans JM, Daly TR: The radiodensity of medications seen on x-ray films . Mayo Clin Proc. 1998, 73:516-9. 10.4065/73.6.516

8. Commandeur JP, Metwaly A, Büchler L, Speiser J, Brander L, Reintam Blaser A: A degrading potassium tablet mimicking active gastric bleeding in a computer tomographic investigation. Case Rep Radiol. 2020, 2020:9791519. 10.1155/2020/9791519

9. Gayer G, Petrovitch I, Jeffrey RB: Foreign objects encountered in the abdominal cavity at CT . Radiographics. 2011, 31:409-28. 10.1148/rg.312105123 